\title{
ANÁlisis COMPARATIVO
}

\section{DE EFICIENCIA EN MERCADOS}

EMERGENTES. El CASO

de Colombia, Chile y Perú

\section{Comparative ANALYSIS}

OF EFFICIENCY IN EMERGING

MARKETS. THE CASE OF

Colombia, Chile and Peru

\author{
Luis Ángel Meneses Cerón ${ }^{\mathrm{I}}$ \\ Camilo Andrés Pérez Pacheco ${ }^{2}$
}

1 Magíster en Administración. Universidad Cooperativa de Colombia. Cauca, Colombia. Correo electrónico: luis.menesesc@campusucc.edu.co Orcid: https://orcid.org/0000-0003-0467-8970

2 Especialista en Gestión Pública. Corporación Universitaria Autónoma del Cauca. Cauca, Colombia. Correo electrónico: camilo.perez.p@uniautonoma.edu.co Orcid: https://orcid.org/0000-0002-3537-8877

Código JeL: G1, G14

Fecha de recepción: 07/05/2019

Fecha de aceptación: 06/06/2019

DOI: https://doi.org/10.18601/16577175.n26.02 


\section{RESUMEN}

El estudio de los mercados bursátiles constituye un tema prioritario en el contexto actual, dada su contribución al desarrollo económico y financiero al facilitar la transferencia de recursos del ahorro hacia la inversión. En ese orden de ideas, el presente artículo analiza comparativamente la hipótesis de eficiencia del mercado en la forma débil de los índices bursátiles de Colombia, Chile y Perú por medio de la aplicación de cuatro pruebas estadísticas a las series de rendimientos en el periodo 2008-2014. Se encontró que los retornos de los tres mercados presentan raíz unitaria, mostrando la presencia de eficiencia informacional en el sentido débil. Lo anterior implica que no es posible para ningún agente económico realizar predicciones sobre los precios de las acciones transadas en ellos, utilizando únicamente información histórica de las cotizaciones.

Palabras clave: hipótesis de eficiencia, caminata aleatoria, prueba de raíz unitaria, mercado emergente, Mercado Integrado Latinoamericano (MILA).

\section{Abstract}

The study of stock markets is a priority issue in the current context, given its contribution to economic and financial development by facilitating the transfer of resources from savings to investment. In this order, this article comparatively analyzes the hypothesis of market efficiency in the weak form of stock indices in Colombia, Chile and Peru through the application of 4 statistical tests to the series of yields in the period 2008-2014. It was found that the returns of the three markets have a unit root, evidencing the presence of informational efficiency in the weak sense. The foregoing implies, that it is not possible for any economic agent to make predictions about the prices of the shares traded in them, using only historical information of the quotes.

Keywords: Efficiency hypothesis, random walk, unit root test, emerging market, MILA.

\section{INTRODUCCIÓN}

En años recientes, la hipótesis de eficiencia del mercado ha sido objeto de análisis en los mercados emergentes, donde algunos de ellos se caracterizan por una reducida diversidad de títulos y relativa baja liquidez. Lo anterior genera una coyuntura atractiva para nuevos inversionistas buscando rendimientos extraordinarios a través de procesos de especulación. No obstante, el cumplimiento de la hipótesis es premisa fundamental de que los precios de los activos incorporan rápidamente toda la información relevante (Zablotsky, 2002), lo que implica la imposibilidad de la obtención de retornos por encima de la media de forma sistemática. 
Fama (1991), en su estudio seminal sobre la hipótesis de eficiencia del mercado, menciona que esta se puede identificar bajo tres escenarios específicos: débil, semifuerte y fuerte. Esto implica que las valoraciones del mercado reflejan la información relevante disponible y, a su vez, se ajustan de forma inmediata a la nueva información, siendo así: las cotizaciones bursátiles que siguen un comportamiento de caminata aleatoria, donde los sucesivos cambios en las valoraciones son estadísticamente independientes con correlaciones seriales iguales a cero.

De esta manera, el modelo de caminata aleatoria desempeña un papel fundamental en el estudio de los activos financieros, puesto que permite identificar si las variaciones en los precios de las acciones tienen un comportamiento errático al azar, como afirma Al-Jafari (2013). En consecuencia, los intentos para predecir los movimientos futuros en los precios de las acciones con datos históricos no son de utilidad, pues no es una forma lo suficientemente precisa y anticipada como para obtener ganancias superiores al promedio.

Ahora, dado que, en el 2009, la Bolsa de Valores de Colombia junto con la Bolsa de Valores de Lima y la Bolsa de Comercio de Santiago propiciaron la creación del primer mercado regional para la negociación de títulos de renta variable bajo el nombre de Mercado Integrado Latinoamericano (MILA), cuya integración entró en operación en mayo del 2011, resulta pertinente desde la economía financiera indagar sobre las condiciones de eficiencia. Así, en este artículo, se analiza la forma débil de la hipótesis de eficiencia del mercado por medio de la aplicación de pruebas de raíz unitaria a los retornos bursátiles de los mercados de Chile, Colombia y Perú, a partir de una perspectiva ex-ante y ex-post al MILA, tomando como referencia de manera puntual el periodo 2008-2014, lapso en el que se configuró una coyuntura económica y financiera de gran complejidad (Collazo y Souto, 2018).

Este artículo se divide en cuatro secciones, la segunda sección presenta la revisión de la literatura. La sección tres contiene los aspectos metodológicos y luego se presentan los resultados obtenidos. Finalmente, el documento culmina con las conclusiones.

\section{REVISIÓN DE LA LITERATURA}

La hipótesis de eficiencia del mercado accionario en su forma general, de acuerdo con lo descrito por Fama (1991), sostiene que los precios de las acciones incorporan rápidamente toda la información disponible; de otra manera, intentar predecir el movimiento de los precios a futuro con su información pasada para la construcción de estrategias de inversión no otorgaría ventajas significativas a los agentes en los procesos de trading.

Según el contenido de la información presente en el mercado se pueden hacer tres distinciones de la hipótesis de eficiencia: débil, semifuerte y fuerte. La hipótesis débil afirma que los precios de las acciones reflejan la información básica presente en el mercado, los datos históricos de precios y volúmenes transados, ocasionando 
que un inversionista por medio de análisis técnico, por ejemplo, no pueda predecir el comportamiento a futuro del precio de la acción (Al-Jafari, 2013).

La hipótesis semifuerte establece que los precios de las acciones reflejan toda la información pública presente en el mercado, no solo el valor pasado de las series de precios y volúmenes transados, sino también la información financiera y contable de la empresa, la economía y toda información pública relevante para la valoración de las acciones. Por tanto, el quehacer del análisis fundamental, donde gran parte de las decisiones de inversión se basan en dicha información, se torna obsoleto en términos de la predicción de los movimientos futuros de precios ( $\mathrm{Za}$ blotsky, 2001).

Finalmente, la hipótesis fuerte, reconoce que los precios de las acciones tienen incorporada tanto la información pública antes descrita como la información privada referente a la empresa. Cabe anotar que si el mercado responde a la hipótesis en su forma fuerte de eficiencia también lo será en sus formas semifuerte y débil, pero si se rechaza la hipótesis de eficiencia en su forma débil, se descarta las otras dos posibilidades de eficiencia (Zablotsky, 2001).

De catalogarse el mercado como eficiente en cualquiera de sus definiciones, "una estrategia de inversión pasiva; es decir, comprar y conservar un fondo de inversiones que replique el índice del mercado resultaría óptimo" (Zablotsky, 2001, p. 6). La teoría de la caminata aleatoria esta intrínsecamente relacionada con la hipótesis de eficiencia en el mercado, puesto que los precios poseen este comportamiento aleatorio cuando los mercados son eficientes.

A partir de lo anterior, los análisis de eficiencia informacional de los mercados han tomado relevancia bajo los escenarios de incertidumbre financiera generados en las crisis de los últimos años, es así como a nivel internacional, Borges (2007) presentó los resultados de las pruebas sobre la eficiencia del mercado de forma débil aplicado al índice de precios del mercado de acciones de Lisboa entre enero de 1993 y diciembre del 2006. Al igual que el presente estudio, Borges utilizó pruebas de raíz unitaria para la comprobación de la hipótesis de que el índice de la bolsa sigue un paseo aleatorio. Al respecto, encontró que el índice bursátil portugués se ha ido acercando a una conducta de caminata aleatoria a partir del 2000.

Posteriormente, Al-Jafari (2013) analizó el mercado de capitales en Estambul. Sus hallazgos revelaron que este mercado no sigue el modelo de caminata aleatoria, de esta manera, demostró que dicho mercado es informativamente ineficiente en la forma débil de la hipótesis, implicando que los inversores identifican los rendimientos anormales utilizando datos históricos de precios de acciones y volumen de operaciones.

Entre los primeros estudios en Latinoamérica, Urrutia (1995) no encontró evidencia suficiente a favor de la eficiencia en los mercados de México, Chile y Argentina para el periodo 1975 a 1991, a partir de la aplicación de pruebas de aleatoriedad. Bekaert, Erb, Harvey y Viskanta (1997) obtuvieron resultados similares en el cálculo del índice de la otrora Bolsa de Valores de Medellín, durante el periodo 1987 a 1994. Por otra parte, Ojah y Karemera (1999) aceptaron la hipótesis de 
eficiencia en los mercados de Chile, Argentina, México y Brasil, la década de 1987 a 1997. Igualmente, Delfiner (2002) acreditó la eficiencia relativa de los mercados de Argentina vs. Estados Unidos, en el periodo de 1993 a 1998, divisando cierto nivel de dependencia de los rendimientos de Argentina, pero hace la salvedad que en este último país se pueden obtener rentabilidades suplementarias al incluir la variable comisiones. Consecutivamente, Valdivieso (2004) analizó la eficiencia del mercado de México en el periodo 1994 a 1999, encontrando evidencia que permite rechazar la caminata aleatoria.

Agudelo (2014) analizó el impacto de la liquidez en el mercado accionario colombiano, señalando una relación clave para este análisis al afirmar que existe una conexión estrecha entre liquidez y eficiencia, además, encontró que la fusión de las bolsas de valores mejoró la liquidez en el mercado. De forma similar, Maya y Torres (2005) estudiaron las consecuencias de la unificación de las bolsas en Colombia, llegando a la conclusión que dicha unión fomentó un aumento de la eficiencia del mercado colombiano, pero con un hallazgo particular, el mercado fue macroineficiente, pero microeficiente.

Arroyave y Agudelo (2012) analizaron el rendimiento exdividendo como un indicador de la eficiencia del mercado, llegando a la conclusión de que el mercado colombiano no es eficiente, dado que los precios no alcanzaron a ajustarse a la información plena de dicha variable. Análogamente, Ramírez, Ruiz y Gutiérrez (2015) y Parga (2017) llegaron a la conclusión de que no existe evidencia suficiente que permita sostener que el mercado accionario colombiano es eficiente a la transmisión de la información, puesto que la metodología utilizada rechaza las hipótesis de existencia de canales de retroalimentación informacional.

\section{Metodología}

La presente investigación constituye un caso de aplicación de la teoría financiera corporativa sobre el desenvolvimiento de los rendimientos de los índices bursátiles que inicialmente consolidaron el miLA. Es, en esencia, un estudio de tipo cuantitativo y comparativo con la finalidad de vincular temáticas contemporáneas, como la valoración de activos financieros, la integración financiera regional, los factores de riesgo internacional y la rentabilidad esperada de un inversionista.

De esta manera, para el análisis del mercado accionario se utilizaron los índices de las bolsas de valores calculados respectivamente por cada entidad, en ese orden, se empleó el IPSA para Chile, COLCap para Colombia e IgBvl para Perú, con datos provenientes de la plataforma Bloomberg Profesional Service. Estos índices miden el comportamiento de los precios de los activos transados más líquidos en las bolsas de cada país excluyendo la información relacionada con el pago de dividendos.

La muestra está compuesta por datos diarios, de lunes a viernes, que inician el 2 de enero del 2008 y finalizan el 31 de diciembre del 2014, para un total aproximado de 1820 observaciones por país. Para los días festivos, en Colombia, Chile 
y Perú, se asumirá que los índices conservan su valor inercial, de tal manera que no se presenten lagunas en las series de datos.

Posteriormente, con el fin de contrastar la hipótesis de eficiencia en su forma débil, de acuerdo con Ruiz y Ruiz (2015), se tomarán como referencia las cuatro (4) pruebas estadísticas más relevantes en la literatura financiera, con el fin de contrastar el modelo de caminata aleatoria.

A partir de Giraldo (2006) se conceptualizan las pruebas estadísticas descritas a continuación:

\section{Prueba Dickey-Fuller (DF)}

La prueba Dickey-Fuller (DF) toma como supuesto que la serie se puede aproximar a un proceso autorregresivo de orden uno (AR (1)) mediante tres variantes:

- Media cero.

- Media diferente de cero.

- Tendencia lineal.

Dado lo anterior, se asume que la serie $\left(Y_{t}\right)$ sigue un modelo AR (1) y se procede a transformar el modelo de la siguiente manera:

$$
\begin{aligned}
Y_{t} & =\theta_{1} Y_{t-1}+\varepsilon_{t} \\
Y_{t}-Y_{t-1} & =\left(\theta_{1}-1\right) Y_{t-1}+\varepsilon_{t} \\
\Delta Y_{t} & =\rho Y_{t-1}+\varepsilon_{t}
\end{aligned}
$$

Teniendo en cuenta lo anterior el test en su hipótesis nula contrasta que $H_{0}: \rho=0$, es decir, existe evidencia estadística que permite afirmar la existencia de una raíz unitaria, dado que $\theta_{1}=1$, lo que equivale a que $\rho=0$. La hipótesis alternativa se plantea como $H_{1}: \rho<0$, en la cual se contrasta el hecho de que no existe información suficiente que permita afirmar si la serie en evaluación tenga una raíz unitaria.

\section{Prueba Dickey-Fuller Aumentada (ADF)}

La prueba de Dickey-Fuller Aumentada (ADF) es una versión modificada de la prueba $\mathrm{DF}$, la cual se puede aplicar para un conjunto más amplio y más complejo de modelos de series de tiempo y en la cual no se supone que el término aleatorio de error no esté correlacionado al asumir que la serie se puede aproximar por un proceso AR (1) con las tres variantes ya descritas. A diferencia del test DF, en esta prueba se contrasta aumentando los valores rezagados de la variable dependiente $\Delta Y_{t}$, de esta manera, y de forma análoga, la prueba se contrasta calculando el siguiente modelo: 


$$
\Delta Y_{t}=\rho Y_{t-1}+\alpha_{i} \sum_{i=1}^{m} \Delta Y_{t-1}+\varepsilon_{t}
$$

Dado lo anterior, las hipótesis de contrastación de la prueba ADF permanecen iguales a las de su par, la prueba DF. Siendo la hipótesis nula de la prueba $H_{0}: \rho$ $=0$, es decir, existe evidencia estadística que permite afirmar la existencia de una raíz unitaria, dado que $\theta_{1}=1$, lo que equivale a que $\rho=0$ y la hipótesis alternativa se plantea como $H_{1}: \rho<0$, en la cual, se contrasta el hecho de que no existe información suficiente que permite afirmar que la serie presente una raíz unitaria.

\section{Prueba de Phillips-Perron}

De acuerdo con lo descrito por Phillips y Perron (1988), esta prueba se utiliza en el análisis de series de tiempo para testear la hipótesis nula de que una serie de tiempo es integrada de orden 1, dicho de otro modo, analiza la existencia de evidencia que permita afirmar si la serie sigue una caminata aleatoria.

Al igual que la prueba de ADF, la prueba de Phillips-Perron asume que el proceso de generación de $Y_{t}$ podría seguir un orden superior de autocorrelación que es aprobado en la ecuación de prueba, haciendo que $Y_{t-1}$ sea endógeno al realizar correcciones no paramétricas en el estadístico de prueba y así invalidar la prueba Dickey-Fuller.

Análogamente, a lo descrito en el test ADF, en la hipótesis nula se contrasta la existencia de un proceso de caminata aleatoria, es decir, existe sustento estadístico que permite afirmar la existencia de una raíz unitaria.

\section{Prueba de Kwiatkowski, Phillips, Smichdt y Shin (KPSS)}

Teniendo en cuenta lo descrito por Antunez (2010), La prueba Kpss permite conocer si la serie es fraccionalmente integrada, esto implica que permite probar tanto la hipótesis de la raíz unitaria como la hipótesis de la estacionariedad para una serie de tiempo, por lo cual se pueden distinguir entre series que parecen ser estacionarias, series que parecen tener una raíz unitaria y series que pueden seguir una tendencia estacionaria.

De esta manera, en el test KPss en la hipótesis nula se contrasta la idea de estacionalidad de la serie, dado que, como lo afirman Kwiatkowski, Phillips, Schmidt, y Shin (1992), la serie se expresa como la suma de la tendencia determinista, la caminata aleatoria y el error estacionario.

He aquí la principal diferencia con los anteriores contrastes de raíces unitarias, en la prueba KPSs, la hipótesis nula asume que la serie es estacionaria, de este modo, en caso tal de rechazarse, la serie sigue un proceso de caminata aleatoria. 


\section{Resultados Y Discusión}

En los gráficos 1 y 2, se presenta la evolución diaria de los índices bursátiles en niveles y las variaciones porcentuales de los retornos para los países de la muestra. Los tres países cuentan con mercados bursátiles emergentes con comportamientos similares. En el caso de Colombia y Chile, sus índices presentaron una leve caída a fínales del 2008, esto puede ser explicado por efecto de la crisis internacional que por el momento se vivía, bache que fue superado con una fuerte recuperación durante el 2009; no obstante, en términos generales de los índices siguen una tendencia al alza a largo plazo.

En el caso del mercado bursátil peruano, se observa una fuerte caída durante el mismo lapso, en ese sentido, a priori se puede afirmar que es producto de una relación de interdependencia con el mercado norteamericano, pues en el 2009 entró en vigencia el tratado de libre comercio con dicho país, que por aquel momento afrontaba una crisis financiera severa.

\section{Gráfico 1. Series en niveles de los índices bursátiles}

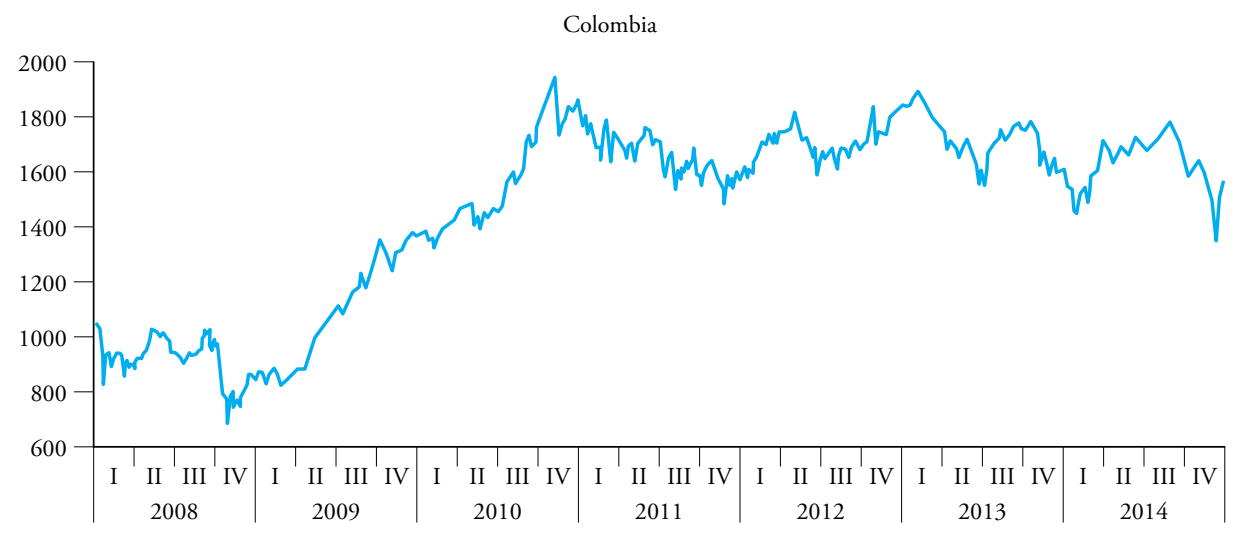

Perú

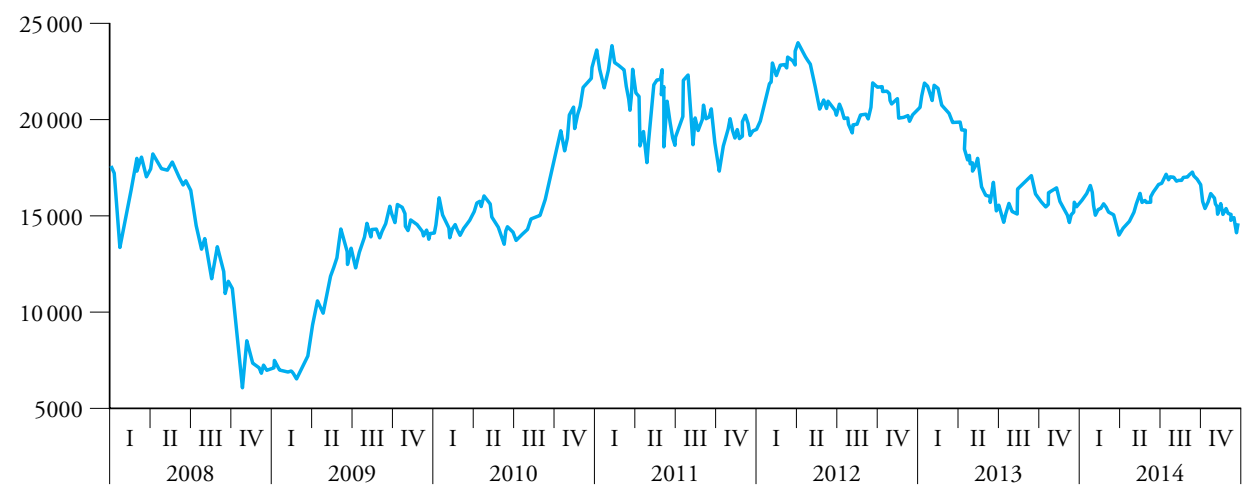




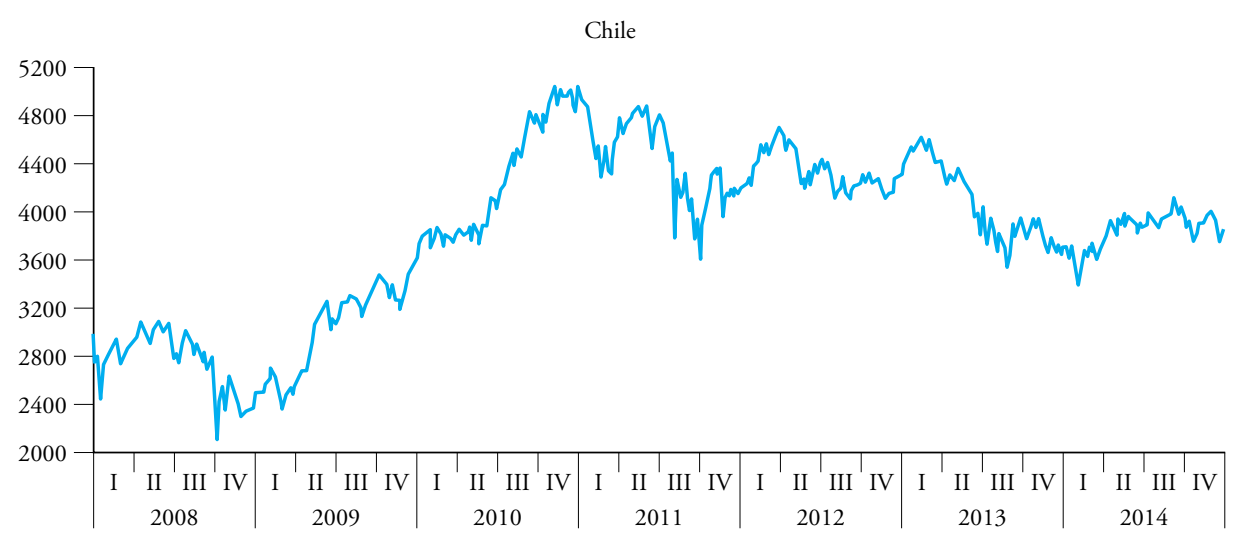

Fuente: elaboración propia con datos de Bloomberg.

Es pertinente resaltar que, tanto en Chile como Colombia, el valor del índice creció al final del periodo estudiado en comparación con el 2008, lo que muestra un notable crecimiento de sus mercados financieros. En general, se observa una senda simétrica histórica de los índices bursátiles de los tres países, desde el 2008 hasta el 2014, lo cual se puede atribuir a la existencia de una interdependencia natural producto de las relaciones comerciales bilaterales (Ruiz, Camargo y Cardona, 2018).

En el gráfico 2, se observa las variaciones porcentuales de los retornos logarítmicos para los tres índices bursátiles en cuestión; esta técnica es usualmente utilizada con el fin de identificar la fluctuación de la varianza en una serie de datos. Durante el tercer trimestre del 2008, se muestra una alta variación de los índices, proceso que se podría imputar a los choques generados por la crisis norteamericana. En el 2011, sucede un proceso similar de menor envergadura, probablemente generado por la instauración del mila. Cabe anotar que dichos picos de volatilidad pueden generar microciclos de turbulencia informativa, congruente con lo identificado por Agudelo (2007).

La tabla 1 condensa las estadísticas descriptivas de las series de los índices bursátiles por países, los valores del sesgo y la curtosis determinan que son muestras sesgadas a la izquierda no normales. Para Colombia y Chile son leptocúrticas, características también típicas de las series financieras. 


\section{Gráfico 2. Variaciones porcentuales de los índices bursátiles}

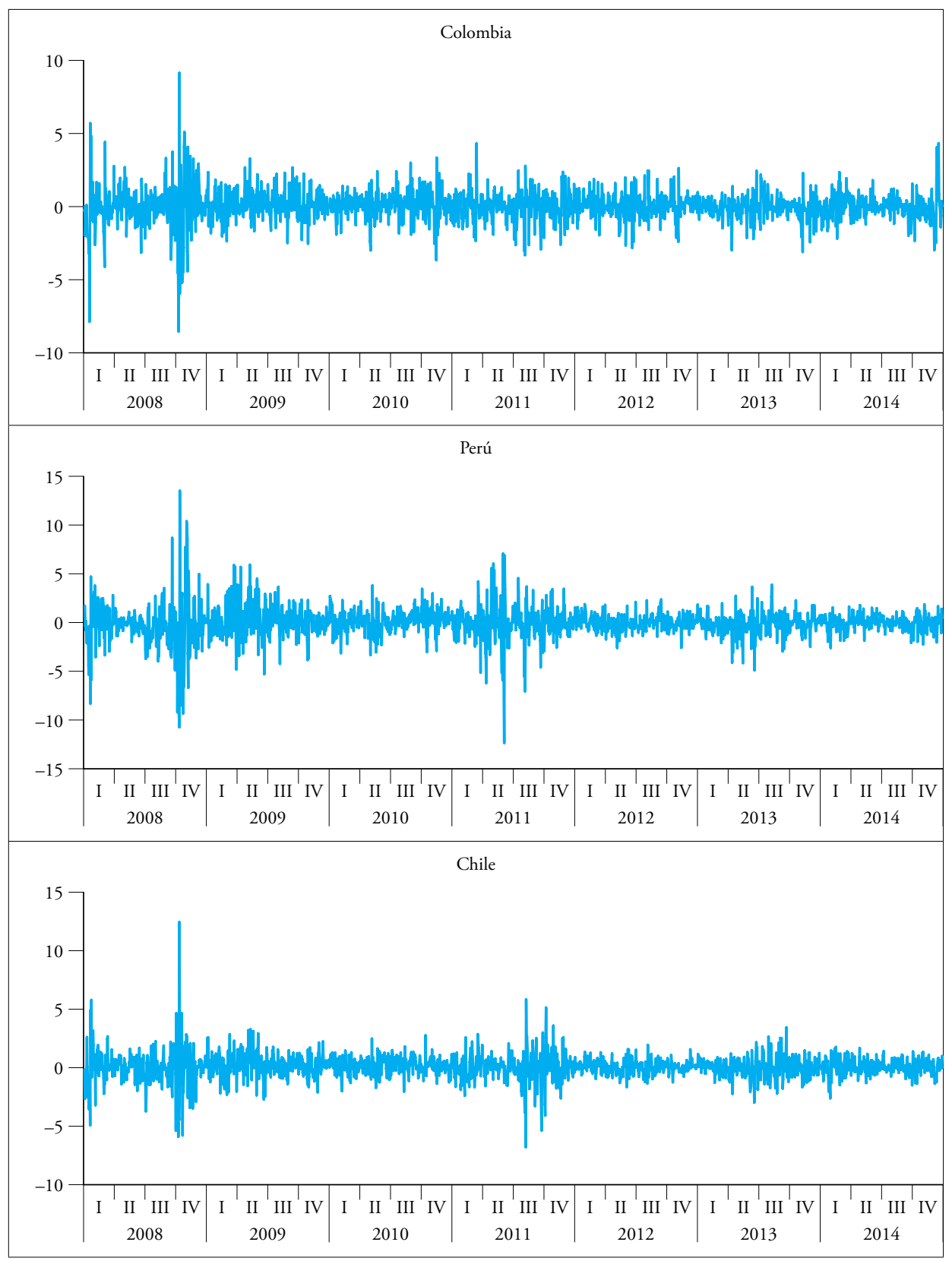

Fuente: elaboración propia con datos de Bloomberg. 
Tabla 1. Estadísticas descriptivas de los índices bursátiles en niveles

\begin{tabular}{|l|r|r|r|}
\hline \multicolumn{4}{|c|}{ Datos para el periodo: enero del 2008-diciembre del 2014 } \\
\hline & \multicolumn{1}{|c|}{ Colombia } & \multicolumn{1}{c|}{ Perú } & \multicolumn{1}{c|}{ Chile } \\
\hline Media & 1472.365 & 16677.29 & 3816.735 \\
\hline Mediana & 1610.950 & 16421.05 & 3898.700 \\
\hline Máximo & 1942.370 & 24051.62 & 5040.970 \\
\hline Mínimo & 686.6400 & 6038.660 & 2101.100 \\
\hline Desviación Estándar & 325.2853 & 3981.939 & 690.0351 \\
\hline Sesgo & -0.861448 & -0.542656 & -0.463713 \\
\hline Curtosis & 2.296772 & $3.132138^{3}$ & 2.323049 \\
\hline Jarque-Bera & 263.4689 & 90.94725 & 100.3068 \\
\hline Probabilidad & 0.000000 & 0.000000 & 0.000000 \\
\hline Observaciones & 1826 & 1826 & 1826 \\
\hline
\end{tabular}

Fuente: elaboración propia.

La tabla 2 contiene los resultados de la aplicación de las pruebas en su orden DickeyFuller aumentada, Dickey-Fuller, estadístico Kwiatkowski-Phillips-Schmidt-Shin (K PPS) y estadístico Phillips-Perron; por medio de los cuales se testeó la existencia de un proceso de caminata aleatoria de forma unánime. Las pruebas Dickey-Fuller aumentada, Dickey-Fuller y estadístico Phillips-Perron analizaron en su hipótesis nula la existencia de raíz unitaria para las series del COLCAP, IPSA y IGBVL a un nivel de confianza del $95 \%$.

Análogamente, el estadístico Kwiatkowski-Phillips-Schmidt-Shin (KPPs), en su hipótesis nula, evaluó la posibilidad de que la serie constatada sea estacionaria. Como se observa, contrastando a un nivel de significancia del $5 \%$ existe evidencia suficiente que permite rechazar la hipótesis nula, en otras palabras, la prueba hace constar que la serie es no estacionaria, siendo este un síntoma característico de los comportamientos de caminata aleatoria.

Los resultados anteriores permiten comprobar que, para los retornos bursátiles de los mercados de Chile, Colombia y Perú, desde una perspectiva ex-ante y expost a la conformación del MILA, tomando como referencia el periodo 2008-2014, existe la condición de eficiencia informacional en la forma débil. 


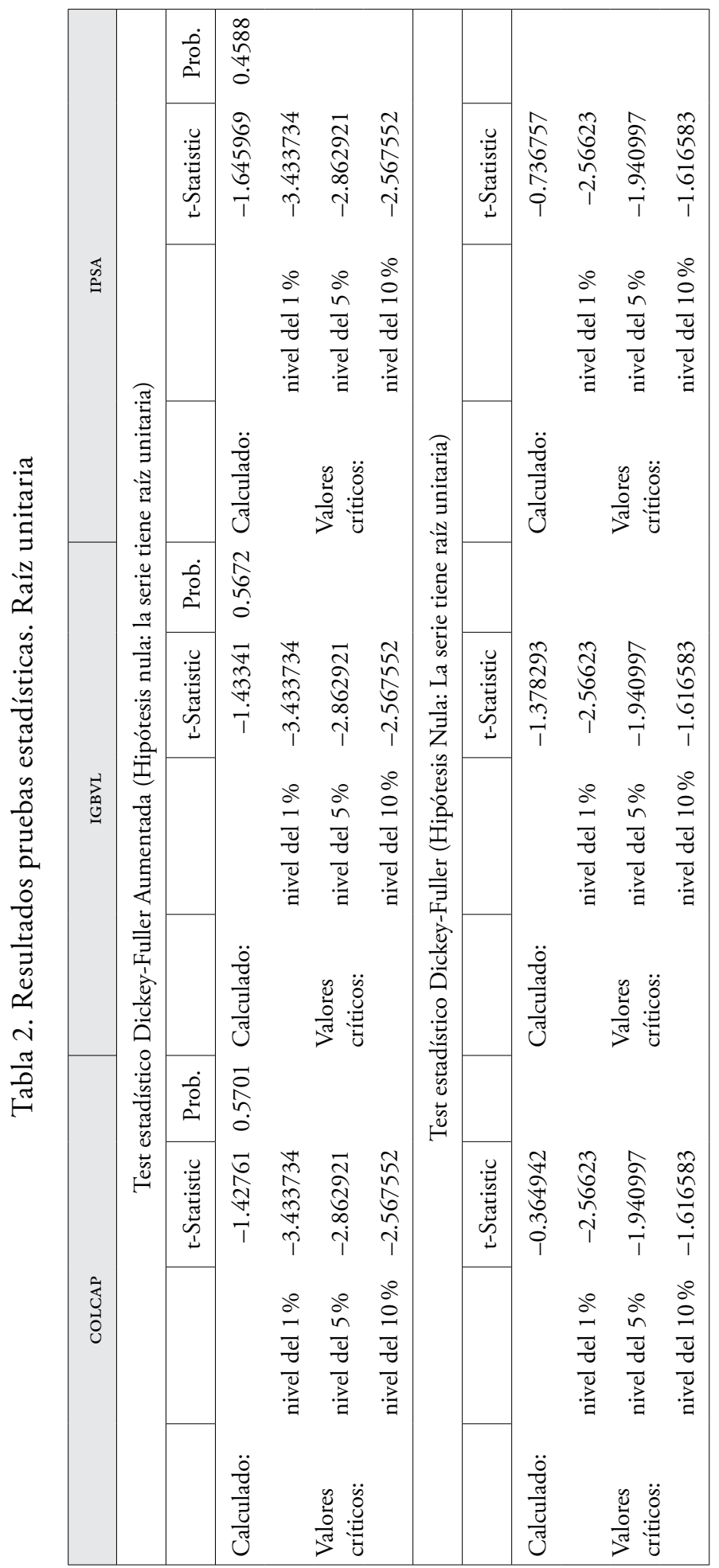

Apuntes Contables n. 26 - Julio-diciembre de 2020 - Pp. 9-24 


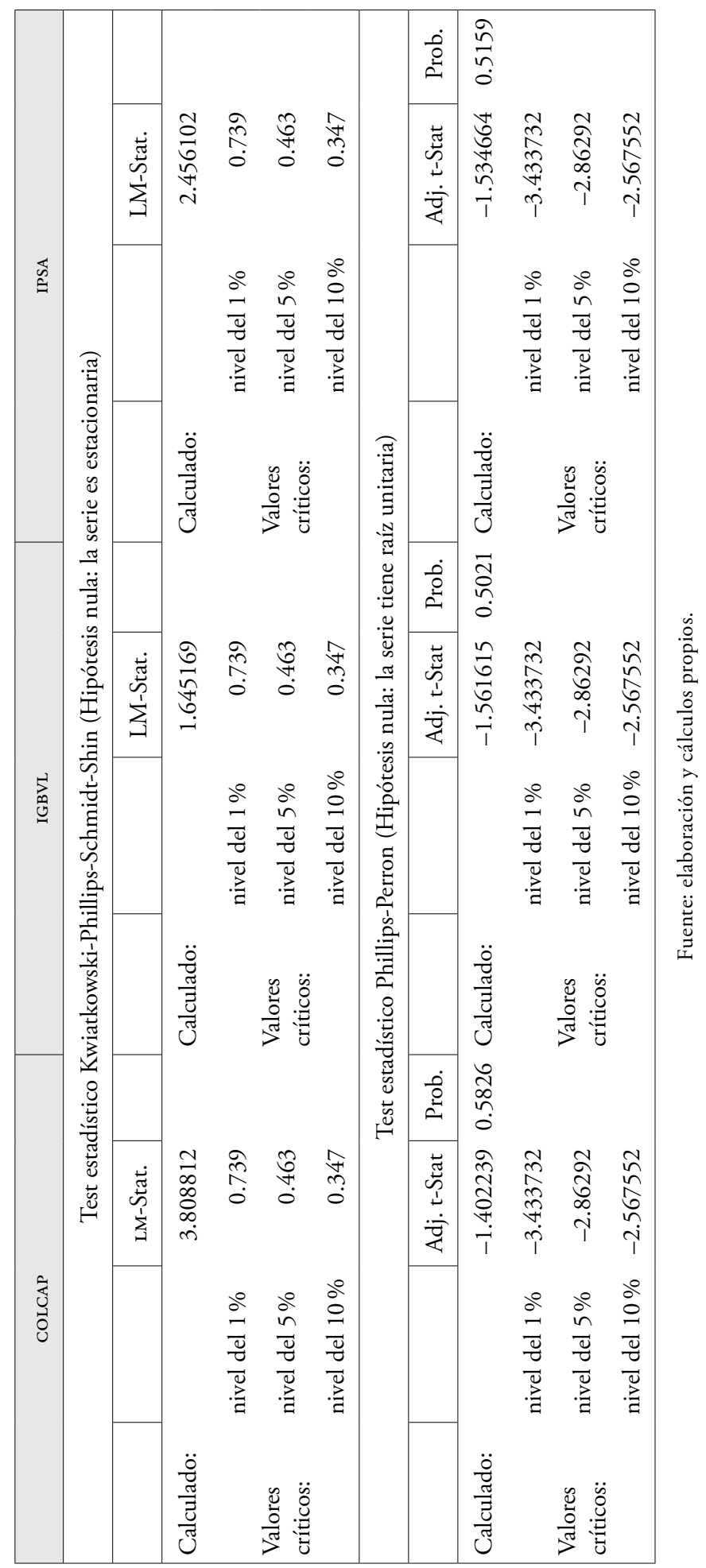




\section{Conclusiones}

Los resultados obtenidos por Arroyave y Agudelo (2012); Ramírez et al. (2015) y Parga (2017), no son congruentes con los expuestos en este estudio, pues cabe anotar que dichas investigaciones analizan periodos de estudio distintos, en los cuales, tentativamente, se pudieron presentar procesos de ineficiencia informacional, teniendo en cuenta que este fenómeno es caracterizado por presentarse en breves periodos. En el caso de Maya y Torres (2004), las series de datos utilizadas son discutibles porque no reflejan el comportamiento real del mercado. Lo anterior, implica que, en cuanto a la revisión de la literatura para los mercados en estudio, los resultados obtenidos son mixtos en la aceptación o el rechazo de la hipótesis de eficiencia, pues, como lo afirma Fama (1991) y Al-Jafari (2013), los fenómenos ineficientes de información son de corto plazo; sin embargo, los mercados tienden a converger hacia una autorregulación, además, los trabajos para Latinoamérica se han caracterizado por no presentar uniformidad en los datos utilizados, dado que, al ser mercados emergentes, sus indicadores base se han ido modificando con el tiempo en contenido y cálculo.

Dada la evidencia empírica sobre el análisis de la forma débil de eficiencia del mercado, usando las pruebas estadísticas Dickey-Fuller aumentada, Dickey-Fuller, estadístico Kwiatkowski-Phillips-Schmidt-Shin (KРPS) y estadístico Phillips-Perron, se determinó que los mercados bursátiles de Chile, Colombia y Perú presentan raíz unitaria, fenómeno característico en un comportamiento de caminata aleatoria, lo que implica que se puede constatar que existen las condiciones básicas de eficiencia informacional durante dicho periodo. Lo anterior significa, entre otras cosas, que no es posible para ningún agente en el mercado realizar predicciones sobre los precios de las acciones transadas, utilizando únicamente información pasada de los precios como es usual en la disciplina del análisis técnico bursátil.

Los hallazgos del estudio son análogos a los resultados expuestos por Sierra, Duarte y Rueda (2015) donde afirman que los índices accionarios de Colombia presentan periodos de alta incertidumbre, por lo que asumir a priori una postura frente a la eficiencia del mercado colombiano resulta inútil. De esta manera, y de acuerdo con Zablotsky (2001), en los mercados con eficiencia informacional, la mejor estrategia de inversión es replicar el índice del mercado o tener un portafolio diversificado incluyendo títulos con bajas correlaciones entre ellos. En este escenario, se reducen los factores de riesgo asociado a la especulación por parte de los agentes presentes en el mercado y desincentiva la atracción de capitales especulativos internacionales en la búsqueda de rentabilidades extras a las determinadas por los mercados. 


\section{REFERENCIAS BIBLIOGRÁFICAS}

Agudelo, D. (2014). Liquid funds in Colombian stock market: How have we improved in the last 10 years. Center for Research in Economics and Finance, 10(8), 1-42. doi: http://dx.doi.org/10.2139/ssrn.2427493

Al-Jafari, M. (2013). Random walk behavior and weak-form efficiency of the Istanbul stock market 1997-2011: Empirical evidence. International Journal of Management, 30(3), 169-199. Obtenido de http://connection.ebscohost.com/c/articles/89146028/ random-walk-behavior-weak-form-efficiency-istanbul-stock-market-1997-2011-empirical-evidence

Antunez, C. (2010). Pruebas de raices unitarias en Eviews. Lima: Manuscrito notas de clase. Arroyave, E. y Agudelo, D. (2012). Ex-dividend return as indicator of efficiency in a emerging stock market: The case of the Colombian stock market 1999-2007. Journal of Economics Finance and Administrative Science, 17(33), 38-47. doi: https://doi. org/10.1016/S2077-1886(12)70006-9

Bekaert, G., Erb, C., Harvey, C. y Viskanta, T. (1997). What matters for emerging markets investments? Emerging markets quarterly. Emerging Markets Quarterly, 17-46. Obtenido de https://www0.gsb.columbia.edu/faculty/gbekaert/PDF_Papers/ what_matters.pdf

Borges, M. (2007). Random walk tests for the Lisbon stock market. IDEAS, 1-19. Obtenido de https://depeco.iseg.ulisboa.pt/wp/wp142007.pdf

Collazo, M. y Souto, L. (2018). Proceso de formación de precios de commodities en las empresas de comercio exterior. Apuntes Contables, 23-41. doi: https://doi. org/10.18601/16577175.n21.03.

Delfiner, M. (2002). Comportamiento de los precios de las acciones en el mercado bursátil argentino, un estudio comparativo. Econstor, (215), 1-29. Obtenido de https://www. econstor.eu/bitstream/10419/84325/1/350461341.pdf

Fama, E. (1991). Efficient capital markets. The Journal of Finance, 46(5), 1575-1617. doi: $10.2307 / 2328565$

Giraldo, N. (2006). Series de tiempo con R. Medellín: Universidad Nacional de Colombia. Obtenido de http://www.medellin.unal.edu.co/ ndgirald/Archivos\%20Lectura/ Archivos\%20curso\%20Series\%20eio/Notas\%20de\%20Clase.\%20Series $\% 20$ de\%20Tiempo\%20con\%20R.pdf

Kwiatkowski, D., Phillips, P., Schmidt a, P. y Shin, Y. (1992). Testing the null hypothesis of stationarity against the alternative of a unit root. Journal of Econometrics, 54, 159178. Obtenido de http://debis.deu.edu.tr/userweb//onder.hanedar/dosyalar/kpss.pdf

Maya, C. y Torres, G. (2005). La unificación del mercado accionario colombiano: un paso hacia la eficiencia. Evidencia empirica. Manizales: Congreso Iberoamericano de Investigación en Administración.

Ojah, K. y Karemera, D. (1999). Random walks and market efficiency tests of Latin American emerging equity markets: A revisit. Financial Review, 34(2), 57-72. doi: 10.1111/j.1540-6288.1999.tb00454.x 
Parga, D. (2017). Evaluación de la hipótesis del mercado eficiente. Bogotá: Institución Universitaria Politécnico Gran Colombiano. Obtenido de http://repository.poligran.edu.co/bitstream/handle/10823/1018/TG-EMA-DIEGO\%2020170802\%20vF. PDF? sequence $=1$ \&isAllowed $=y$

Phillips, P. y Perron, P. (1988). Testing for a unit root in time series regression. Oxford University Press, 75(2), 335-346. doi: 10.2307/2336182

Ramírez, J., Ruiz, E. y Gutiérrez, B. (2015). Hipótesis de mercados eficientes en el mercado accionario colombiano a través del estudio de eventos. xx Congreso Internacional de Contaduría, Administración e Informática (pp. 1-18). México: unam. Obtenido de http://congreso.investiga.fca.unam.mx/docs/xx/docs/13.03.pdf

Ruiz, A. y Ruiz, B. (2015). The efficient-market hypotesis and the modelling of mexican stock series: a multivariate analysis. Economia Informa, 390, 28-57. doi: https:// doi.org/10.1016/S0185-0849(15)30003-7

Ruiz, L., Camargo, D. y Cardona, O. (2018). Relación entre rentabilidad y adhesión al pacto global: un análisis de empresas colombianas. Apuntes Contables, 156-166. doi: https://doi.org/10.18601/16577175.n22.10.

Urrutia, J. (1995). Tests of random walk and market efficiency for latin american emerging equity markets. Journal of Financial Research, 18(3), 299-309. doi: https://doi. org/10.1111/j.1475-6803.1995.tb00568.x

Valdivieso, R. (2004). Validación de la eficiencia y modelos de fijación de precios en el mercado mexicano de valores. México, CDMx: Universidad Nacional Autónoma México.

Zablotsky, E. (2001). Eficiencia del mercado de capitales. Una ilustración. IDEAS, (194), 1-41. Obtenido de https:/ucema.edu.ar/publicaciones/download/documentos/194.pdf

Zablotsky, E. (2002). Consideraciones sobre la eficiencia del mercado de. Econstor, (213), 1-35. Obtenido de https://www.econstor.eu/bitstream/10419/84299/1/346327504.pdf 